\title{
Regulación de la salud en Colombia: un problema de información secuestrada*
}

\author{
Health Regulation in Colombia: \\ A Problem of Captured Information
}

\author{
Alejandro Pérez y Soto Domínguez ${ }^{* *}$ \\ Katherine Flórez Pinilla*** \\ Fernando Giraldo García ${ }^{* * * *}$
}

Recibido: 29 de abril de 2015

Aprobado: 12 de febrero de 2016

Disponible en línea: 30 de enero de 2017

\section{Resumen}

Desde la teoría económica austriaca se identificaron problemas en el modelo teórico de asistencia sanitaria conocido como managed competition aplicado en Colombia bajo la Ley 100/1993. Estos problemas encuentran relación con la crisis que afronta el sistema de salud en Colombia en la actualidad. El objetivo de este artículo es, por tanto, analizar el fundamento teórico y las razones de estas fallas. Para su desarrollo, se utilizó un enfoque de metateoría en el cual se estudiaron los supuestos de una teoría (managed competition) desde otra (austriaca)

\begin{abstract}
Based on Austrian Economic Theory, problems were identified in the Health Care Model, known as "Managed competition", related to the current crisis in the Health Care System in Colombia. The objective of this article is to analyze the theoretical basis and reasons for such failures. For its development a meta-theoretical approach was used in which the assumptions of a theory (managed competition) from another (Austrian) as a form of criticism and feedback needed to improve the theoretical constructions and especially their practical applications in
\end{abstract}

doi:10.11144/Javeriana.papo22-1.rscp

${ }^{*}$ Artículo de investigación

** Economista por la Universidad del Valle, magíster en Economía de la Escuela Austriaca por la Universidad Rey Juan Carlos y doctor en Ciencias Económicas por la Universidad Rey Juan Carlos. Docente investigador de la Universidad del Norte. Correo electrónico: aysoto@uninorte.edu.co Orcid: http://orcid.org/0000-0001-5190-5297

*** Economista por la Universidad Industrial de Santander, magíster en Derecho Económico por la Universidad Externado de Colombia y doctoranda en Law-Business Administration en la Universitat de Lleida. Docente investigadora de la Universidad San Buenaventura de Cali. Correo electrónico: kflorez1@usbcali.edu.co Orcid: http://orcid.org/0000-0002-6966-2075

**** Diploma Ciencias Políticas por el Institut des Hautes Etudes de l'Amérique latine, magíster en Ciencias Políticas Sociedades Latinoamerianas por la Université Sorbonne Nouvelle - Paris 3 y doctor en Ciencia Política por la misma universidad. Docente investigador de la Universidad del Norte. Correo electrónico: fernandogiraldo@ uninorte.edu.co Orcid: http://orcid.org/0000-0002-2841-9298 
como forma de crítica y retroalimentación necesaria para mejorar las construcciones teóricas y especialmente sus aplicaciones prácticas en el ámbito de las políticas públicas. Los resultados de los análisis demuestran que los problemas corresponden a inadecuados supuestos metodológicos sobre el comportamiento de los agentes del mercado que impiden la coordinación, la competitividad y la eficiencia, en este caso de las empresas aseguradoras y prestadoras del servicio. Dichos supuestos metodológicos se dividen en tres: 1) antropológicos, 2) sobre la relación hombre-entorno y 3) sobre la regulación. A partir de estos tres supuestos, se identificó la existencia de información secuestrada y cuatro problemas regulatorios derivados: 1) organización estática de los agentes en la infraestructura del sistema, 2) regulador parcializado, 3) segmentación y no correspondencia del producto con la demanda y 4) discriminación de precios por capacidad de pago: selección adversa, corrupción y free riders. Se concluye que un cambio en el sistema de salud demanda un diseño institucional basado en supuestos más cercanos al comportamiento de agentes del sistema.

\section{Palabras clave:}

regulación; supuestos metodológicos; supuestos epistemológicos; economía de la salud; sistema de salud colombiano

\footnotetext{
Cómo citar este artículo:

Pérez y Soto Domínguez, A., Flórez Pinilla, K. y Giraldo-García, F. (2017). Regulación de la salud en Colombia: un problema de información secuestrada. Papel Político, 22(1), 105-125. https:// doi.org/10.11144/Javeriana.papo22-1.rscp
}

the field of public policy. The article shows that these problems are derived from inadequate methodological assumptions that impede the coordination, competitiveness and efficiency of the service-providing companies. These methodological assumptions are divided into three: anthropological assumptions, assumptions about the man-environment relationship and assumptions about regulation. From these three assumptions, the existence of kidnaped or captured information and four regulatory problems were identified: 1) Static organization of the agents in the system infrastructure; 2) Biased regulator; 3) Segmentation and noncorrespondence of product with demand; and 4) Discrimination of prices by payment capacity: adverse selection, corruption and free riders. It is concluded that a change in the health system demands an institutional design based on assumptions closer to the behavior of the agents of the system.

\section{Keywords:}

regulation; methodological assumptions; epistemological assumptions; health economic; Colombian health system 
Es un error creer que, a la larga, la mejor y más barata manera de alcanzar cualquier objetivo consiste en someterse a un plan previo en lugar de utilizar en cada momento los medios disponibles más idóneos... El principio de que los monopolios estatales se corrompen con el transcurso del tiempo es de tanta aplicación a este caso como a cualquier otro.

Hayek

\section{Introducción al problema teórico-organizativo del sistema de salud colombiano}

Las últimas reformas en el sistema de salud colombiano no solo muestran la grave crisis financiera y de legitimidad en que este se encuentra, sino además la falta de formas regulatorias alternativas que cambien la organización del sistema actual, de tal manera que solucionen los problemas en él evidenciados, sino que, además, promuevan un sistema de salud eficiente en el manejo de los recursos, efectivo en la atención, la solución y la prevención de enfermedades y competitivo entre los actores que lo conforman como medio para el avance tecnológico del sistema y la reducción constante de costos.

Sin embargo, no hay una única respuesta que dé cuenta del alcance de dicha eficiencia, efectividad y competencia. En la actualidad, sigue siendo tema de debate el modo de provisionar asistencia sanitaria en los países y la oportunidad de los implementados hasta el momento. Los métodos hasta ahora conocidos son los siguientes: 1) modelo de provisión y financiación pública estatal directa, 2) el modelo de provisión y financiación privada (Flórez, 2008, 2010) y 3) algunos modelos mixtos de provisión y financiación. La elección de uno u otro modo se convierte en el centro de deliberación pública, aún más cuando de entrada se rechaza el sistema de salud privado, asumiéndose que la provisión privada significa exclusión de gran parte de la población del acceso a servicios o la inclusión de todos sacrificando la atención satisfactoria al usuario: "la calidad y la humanización” del servicio (Calderón, 2015, p. 51); este último un problema constante en el sistema de salud colombiano (Matajira, Escobar y Barney, 2016).

A pesar de las creencias generalizadas en esta incompatibilidad, diversas perspectivas económicas han demostrado la oportunidad del modo privado para garantizar bienes como la asistencia sanitaria que, por sus características de bien escaso como cualquier otro, requiere recursos también escasos para proveerla, aunque muchos se nieguen a aceptarlo $^{1} \mathrm{y}$, por tanto, sea preciso garantizar su eficiencia: "Requiere de una función

\footnotetext{
${ }^{1}$ Desde la misma teoría se presume, por ejemplo, que el trabajo del médico no busca remuneración, por lo cual el mercado resulta un inadecuado distribuidor de recursos: "La búsqueda del beneficio no es una explicación adecuada de la actividad en el 'mercado' médico. La atención en los hospitales a través de los siglos ha sido primordialmente pública o bajo el auspicio de instituciones sin ánimo de lucro. Aun cuando los servicios médicos en Estados Unidos están organizados sobre bases tan individualistas como cualquier profesión o empresa, los
} 
de producción que combine los recursos de un modo eficiente a fin de obtener el mayor impacto social, y que sirva de instrumento para alcanzar la equidad" (Collazo et al., 2002, p. 359). Estas perspectivas han valorado más la teorización privada que la pública, porque han sido más graves las fallas de Estado que las fallas del mercado como medio para alcanzar la eficiencia. Teóricamente, se ha recomendado como el mejor sistema el de mercado, siempre y cuando sus fallas sean corregidas por un regulador imparcial, papel que ha asumido el Estado hasta la actualidad.

Los últimos cincuenta años han sido testigo de los matices que pueden tener los dos modelos extremos y de sus combinaciones cuando se delega al Estado el diseño del sistema. Desde el punto de vista del modelo de financiamiento público de la salud, Europa es el mejor ejemplo de provisión estatal directa. En un ranking elaborado por Health Consumer Powerhouse (2016), Holanda fue calificado como el mejor sistema de salud, seguido de Suiza, Noruega, Finlandia y Dinamarca. Sin embargo, se dice que los sistemas de salud del mundo han llegado a puntos de insostenibilidad financiera, al pretender separar el servicio de la capacidad de pago.

Comparando Colombia con el precio total de cada tipo de sistemas en términos corrientes, se obtuvo un ranking que nos permite aproximarnos a las capacidades de producción de un sistema de salud eficiente, equitativo y universal, teniendo en cuenta el indicador usual del gasto en salud como porcentaje del PIB. ${ }^{2}$ Encontramos que en materia de financiamiento, para tener un sistema de salud como el noruego o el suizo, en términos nominales, Colombia necesitaría disponer de 117 \% de su PIB; para uno como el de Luxemburgo, 115 \%; el de los Estados Unidos 113 \%; el de Mónaco, 110 \%; y el canadiense, $76 \%$. Si comparamos con la situación actual, nuestro país destina aproximadamente 7 \% del PIB en salud, cerca de países como Kazajistán, Surinam, Paraguay y Ecuador (Banco Mundial, 2011). “El gasto sanitario mundial se aproxima a US\$ 5,3 billones al año. Los países de la OCDE solo representan el 18 \% de la población mundial, pero constituyen el 86 \% del gasto sanitario del mundo" (OPS/OMS, 2011, p. 18).

El gasto público en salud en los diez países con mayor índice de desarrollo humano es proporcionalmente mayor que el privado, excepto en los Estados Unidos. En estos países, 70 \% o más del gasto es público. Esta relación se invierte paradójicamente en la mayoría de los países con desarrollo humano bajo. La OPS/OMS estima que 150 millones de personas sufren catástrofes financieras por la enfermedad y el pago de los

médicos han aceptado su papel social en la comunidad y frecuentemente han atendido la enfermedad y promovido la salud sin remuneración por su tiempo y sus conocimientos" (Mushkin, 1999, pp. 94-95).

2 "Hay una tendencia a medir el gasto en salud como proporción del PIB, pero ello es obviamente insuficiente porque las diferencias en los valores absolutos impactan en las capacidades de oferta general de los servicios de salud, crecimiento económico y cobertura de la salud de las personas están íntimamente correlacionados, los gastos de salud de los hogares aumentan conjuntamente con el incremento de los ingresos. Esto tiene justificación porque las personas, cuando pueden, prefieren vivir más, pero, además, saludablemente" (OPS/OMS, 2011, p. 17). 
servicios médicos, 100 millones de personas caen en la pobreza y más de $50 \%$ de la población carece de protección formal en salud. En algunos países, 11 \% de la población tiene dificultad financiera grave y $5 \%$ de la población cae en la pobreza por este motivo (2011, p. 32).

En países como Colombia, clasificados por su PIB como de ingreso medio-alto y puesto 84 en el mundo, el gasto en salud ${ }^{3}$ se situó en 2014 alrededor de 7 \% del PIB. Un sistema de salud financiado completamente por el Estado parece insostenible en Colombia con la capacidad económica de la nación para generar excedentes (figura 1).

Figura 1. Gasto en salud en Colombia (\% del PIB).

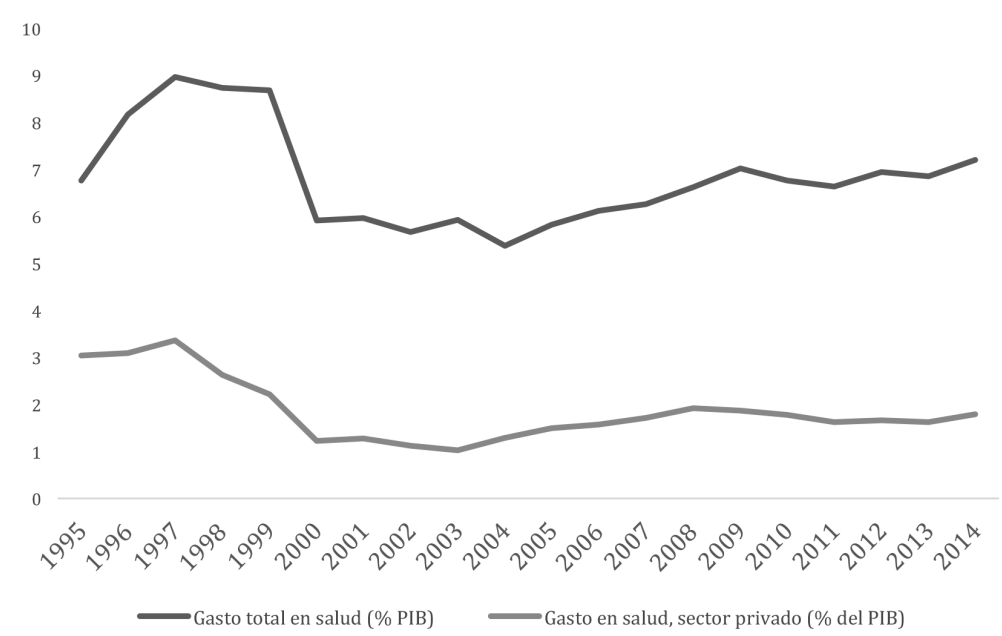

Fuente: Banco Mundial (2015).

Las críticas a los fallidos sistemas de salud recaen sobre las formas de organización de los sistemas de salud, el modo de financiamiento y los resultados en materia de cobertura y calidad. No obstante, estas críticas son contradictorias:

De una parte, se dice que el sistema es demasiado oneroso y, por otra parte, que no hay suficiente inversión. Las autoridades reclaman que los costos de salud aumentan más que el crecimiento económico lo cual provoca endeudamiento. Pero, simultáneamente, se critica a los servicios públicos por no otorgar servicios de calidad [lo cual aún torna indefinido el debate]. (OPS/OMS, 2011, p. 18).

${ }^{3}$ Según el Banco Mundial (2015), el gasto total en salud es la suma del gasto público y privado en salud. 
Ante las problemáticas de la organización y la financiación de la asistencia sanitaria, la economía ha realizado desarrollos teóricos (Arrow, 1963), y se ha especializado en temas como la organización de los agentes del sistema y los incentivos para la actuación de cada agente del mercado de la asistencia. En Colombia, el principal referente teórico del actual diseño del sistema de asistencia sanitaria que inspiró la Ley 100/1993 fue Enthoven (1993), con su modelo de managed competition y sus defensores de la época en el Banco Interamericano de Desarrollo (Londoño y Frenk, 1997). Antes de la Ley 100, el país había adoptado formas difusas de organización siguiendo tendencias mundiales sobre el tema (tabla 1); sin embargo, hace un poco más de dos décadas la organización política del país le ha apostado al modelo de Enthoven como rector del sistema.

Tabla 1. Modelos de salud aplicados en Colombia

\begin{tabular}{|l|l|l|l|}
\hline Periodo & \multicolumn{1}{|c|}{ Modelo de prestación } & Papel del Estado & \multicolumn{1}{c|}{$\begin{array}{c}\text { Ley de intervención o } \\
\text { regulación }\end{array}$} \\
\hline $\begin{array}{l}1886- \\
1936\end{array}$ & $\begin{array}{l}\text { Higienista, asistencia sanitaria } \\
\text { libre y caridad }\end{array}$ & Salud pública & Ley $30 / 1886$ \\
\hline $\begin{array}{l}1937- \\
1975\end{array}$ & Bismarckiano & $\begin{array}{l}\text { Productor parcial } \\
\text { ISS, salud pública }\end{array}$ & $\begin{array}{l}\text { Acto legislativo 01 de } 1936 \\
\text { Ley 96/1938 } \\
\text { Ley 90/1946 }\end{array}$ \\
\hline $\begin{array}{l}1975- \\
1993\end{array}$ & Provisión estatal-Keynes & Productor directo & Decreto 56/1975 \\
1993 & $\begin{array}{l}\text { Competencia regulada-Enthoven: } \\
\text { precio (UPC), producto (POS) y } \\
\text { aseguramiento regulado }\end{array}$ & "Regulador" & $\begin{array}{l}\text { Ley } 100 / 1993, \text { Ley } \\
1122 / 2007 \text { y Ley } 1438 / 2011\end{array}$ \\
\hline $\begin{array}{l}2014- \\
2017\end{array}$ & $\begin{array}{l}\text { Competencia regulada- } \\
\text { Enthoven: eliminación del POS y }\end{array}$ & "Regulador" & $\begin{array}{l}\text { Ley Estatutaria } 1751 / 2015 \text { y } \\
\text { Resolución 2158/2016 }\end{array}$ \\
\hline
\end{tabular}

UPC: unidad de pago por capitación; POS: plan obligatorio de salud;

ISS: Instituto de Seguros Sociales.

Fuente: Elaboración propia.

En la actualidad, el sistema colombiano demanda cambios en sus estructuras y la política pública no le ha apuntado a la evaluación de los fundamentos teóricos de esta. De hecho, afirman que los problemas del sistema son de la práctica, no de la teoría. En este artículo, atenderemos dicha evaluación desde una perspectiva teórica de economía de la regulación. Para esto, manejará dos hipótesis: por un lado, que el modelo de Enthoven no se aplicó correctamente en Colombia; y por otro, que el modelo de Enthoven tiene en sí mismo problemas metodológicos que no son compatibles con el funcionamiento real de un mercado de la salud.

Buena parte de los problemas del sistema de salud colombiano se deben a apreciaciones metodológicas incompletas o inadecuadas: los axiomas que utiliza, la propuesta 
antropológica y la noción de eficiencia paretiana, que no solo han sido criticados en el ámbito del mercado de salud, sino en la teoría económica en general. Se reclama, entonces, que se retome el debate teórico y se amplíen las reformulaciones en el área.

Este artículo tomará posición para abordar la problemática de los sistemas de salud desde la escuela austriaca, en primer lugar, haciendo uso de sus conceptos para contrastar el modelo actual con un modelo ideal con atributos tales como la eficiencia, efectividad y competencia mencionados arriba; y en segundo lugar, identificar los problemas en la fundamentación teórica que puedan estar incentivando las conductas nocivas para el funcionamiento del sistema. Finalmente, se propondrá una forma de ver el problema que lleve a trasformaciones del modelo colombiano.

\section{Revisión del modelo organizativo actual}

Desde un marco teórico basado en la escuela austriaca de economía, se pueden adoptar criterios para evaluar tipos de teorías y regulaciones; por consiguiente, deducir el origen y las consecuencias de la intervención política. Los criterios metodológicos se dividen en tres: 1) criterios sobre el hombre que hemos denominado supuestos antropológicos, 2) supuestos sobre la relación hombre-entorno que se dividen a su vez en el tiempo y la información y 3) necesidades institucionales como respuesta a las condiciones anteriores o los "márgenes de acción que tienen las instituciones para procurarlo, proveerlo o impedirlo" (Pérez y Soto, 2013) por medio de intervenciones políticas (tabla 2).

Tabla 2. Fundamentos metodológicos de la teoría económica de la salud: propuesta para una evaluación

\begin{tabular}{|l|l|l|}
\hline $\begin{array}{c}\text { Supuesto } \\
\text { metodológico }\end{array}$ & $\begin{array}{c}\text { Fundamento neoclásico } \\
\text { "renovado" de Arrow a } \\
\text { Enthoven, economía de la salud }\end{array}$ & \multicolumn{1}{|c|}{$\begin{array}{c}\text { Fundamento austriaco, } \\
\text { alternativa opuesta }\end{array}$} \\
\hline Hombre & $\begin{array}{l}\text { Homo œconomicus Aislado, } \\
\text { racional, maximizador y egoísta }\end{array}$ & $\begin{array}{l}\text { Producto social y cultural, responde } \\
\text { tanto a racionalidad instrumental como } \\
\text { a códigos de conducta, principios } \\
\text { morales y demás convenciones } \\
\text { adoptadas en su marco social }\end{array}$ \\
\hline Tiempo & No existe, análisis estático & Dinámico, análisis evolutivo \\
\hline Información & $\begin{array}{l}\text { Asimétrica entre los agentes del } \\
\text { sistema }\end{array}$ & $\begin{array}{l}\text { Información y conocimiento tácito, } \\
\text { disperso, subjetivo (Huerta de Soto, } \\
2005)\end{array}$ \\
\hline
\end{tabular}

Fuente: Elaboración propia.

En la teoría de la provisión de salud de Enthoven (1993), se puede identificar el supuesto de hombre económico, enteramente racional y maximizador, una visión estática de este en relación con el tiempo y una concepción limitada sobre el carácter y la influencia de la información sobre el comportamiento del primero. Establecer un 
mercado regulado de la salud evidencia fallas al considerar de forma estática el mercado de la asistencia sanitaria, y no tener en cuenta la importancia de la información y de la conducta empresarial natural de los agentes del mercado, motivada por procesos que combinan racionalidad y prueba-error, por lo cual es evolutiva y dinámica. Un modelo estático no logra acercarse a las conductas naturales de los agentes consumidorproductor-regulador y no tiene en cuenta la naturaleza económica de los bienes que componen el sistema como un todo interrelacionado, por lo cual se puede catalogar como incompleto e inadecuado. Como resultado, tendremos políticas ineficaces, más tendientes a acentuar fallas que a permitir un marco que posibilite el aprovechamiento máximo de los recursos del sistema.

Enthoven modela la posibilidad de un mercado perfectamente competitivo por medio de la existencia de cuatro agentes racionales en el mercado: 1) consumidores de servicios de salud, 2) productores de servicios de salud, 3) intermediarios o aseguradores de salud y 4) un regulador imparcial que define las reglas para la interacción entre los tres primeros y el precio que se debe pagar por determinados servicios que prestan los segundos.

En Colombia, se plasmó esta propuesta en la Ley 100/1993 de la siguiente manera: dos tipos de consumidores, inicialmente desagregados según capacidad de pago: por contribución salarial y por subsidio del Estado (regímenes contributivo y subsidiado); productores denominados instituciones prestadoras de salud (IPS), aseguradores o empresas promotoras de salud (EPS); y regulador: función fragmentada en tres autoridades: Ministerio de Salud y Protección Social (antes Comisión de Regulación en Salud y Consejo Nacional de Seguridad Social en Salud), Fondo de Solidaridad y Garantía y Superintendencia de Salud, con la labor de definir los precios y el producto del mercado modelado de manera periódica, que han denominado unidad de pago por capitación (UPC) y plan obligatorio de salud (POS); estos últimos actualmente destinados a desaparecer después de la nueva reforma al sistema. Sin embargo, la cotización obligatoria a este sigue siendo regulada y los precios de los medicamentos.

Para ofrecer los bienes contenidos (POS y no POS), se organizó el sistema bajo dos tipos de agentes: EPS e IPS, las primeras patrocinadoras o representantes de los afiliados (tanto de consumidores contribuyentes como de subsidiados), las segundas oferentes tanto de naturaleza pública como privada y solidaria (Flórez, 2010a) dedicadas a atender y prevenir la enfermedad, lo cual queda ratificado en el artículo 14 de la Ley 1122/2007. Sin embargo, el modelo a la colombiana distribuyó la función patrocinadora entre la aseguradora y el Estado, contrario a lo propuesto por Enthoven, para quien debía recaer solo en la aseguradora y, por tanto, tener un margen de libertad para diferenciarse y competir por clientes. El Estado representa también al consumidor emitiendo reglas del juego en favor de este para el funcionamiento del mercado, las ya mencionadas reglas de precio y de producto, además de financiación, vigilancia y control de calidad del sistema 
en su conjunto (que incluye el subsector de medicamentos), así como determinar cuáles empresas entran y salen del mercado. De este modo, en Colombia, el modelo recayó en la función reguladora del Estado y así eliminó la posibilidad de autorregulación de los agentes, posible solo en un ambiente competitivo.

En un modelo microeconómico simple y estático, un precio regulado o definido por una autoridad, diferente del fijado por el mercado, distorsiona la distribución eficiente de los recursos. Si se impone un precio por encima del precio de equilibrio, el efecto derivado será un exceso de oferta, mientras que, si el precio es inferior, se dará un exceso de demanda y escasez generalizada del producto. Evidenciamos el segundo problema deduciendo, entonces, que el precio es inferior al posible equilibrio sumando los problemas de demanda inducida connaturales a la asistencia sanitaria. Hay exceso de demanda, con el agravante de que la demanda se "induce" hacia nuevas demandas que no solucionan en muchos casos la necesidad de salud. A diferencia de otros mercados, cuando alguien demanda asistencia sanitaria, demanda con ella otros bienes complementarios: asistencia-medicamentos-intervenciones, pero no conoce cuáles requiere (información asimétrica), por su escaso conocimiento sobre medicina, su cuerpo y el origen de la enfermedad, por tanto, deja esa decisión al médico, el médico a su vez es contratado por la EPS para minimizar costos, inducirá al paciente a una solución económica a corto plazo, por lo cual se ve menguada su libertad-autonomía para inducir una demanda desligada del análisis costo-beneficio de la EPS. Algo que se conoce en la opinión pública como "la cultura del acetaminofén" (El Espectador, 25 febrero 2013).

La relación principal-agente nociva entre las EPS y el médico se ha tratado de menguar mediante las reformas al sistema desde 2007 que ha limitado, entre otras, la integración vertical entre el aseguramiento y la prestación (Ley 1122/2007), y por otro lado, creando protección legal para la autonomía médica (Ley Estatutaria de 2015, art. 17). La integración vertical no era imaginable por el legislador de 1993 si asumía como supuesto el modelo estático de Enthoven: no permitir modelar la dinámica de los agentes impidió que se predijera que los aseguradores, para controlar costos y maximizar ganancias, contratarían (controlarían y vigilarían) a los médicos de consulta externa o primer nivel de atención para inducir o encauzar las demandas del paciente y alinearlas con la función de costos.

Hoy la nueva reforma elimina el POS y la UPC, se propone otorgar todos (con ciertas excepciones) los bienes que demande el paciente y un sistema de información para que el médico no deba contar con la aprobación de su contratante: todos los medicamentos y tratamientos que necesite el paciente. Situación ideal, pero, como contrapeso, para salvaguardar la sanidad financiera del sistema se establecerá la función rectora de regulación de precios de medicamentos. Lo cual inyectará los mismos efectos distorsionadores del mercado de la asistencia sanitaria derivados de los medicamentos. 


\section{Malos supuestos, mala regulación}

Enthoven asume que es posible proveer una regulación que permita surgir la competencia en un mercado que no es competitivo, por medio del manejo total de la información del sistema y del conocimiento de los incentivos de cada agente y especialmente de un regulador: "Armed with data and expert advice, and informed, cost-conscious consumers" (un regulador armado con información, asesoramiento de expertos y consumidores conscientes de los costos en que incurren $)^{4}$ (Enthoven, 1993, p. 29).

La información total del sistema no es posible de obtener y tampoco de prever. Por tanto, no es posible para el regular controlar todas las variables que fomentan la competencia. Los elementos que permiten aflorar la competencia se derivan de los precios y las variedades de productos que puedan brindar las empresas que hagan parte del sistema. Las innovaciones, si son definidas antes de su producción, se asimilarían más a un esquema de planificación central que a uno de mercado, además que no tendría sentido hablar de innovación-planificada, pues esta no se puede anteceder o prever, por lo cual el término managed competition no es adecuado para denominar cualquier modelo competitivo en la provisión de asistencia sanitaria. Al no existir precios ajustados a la espontaneidad de los cambios que pueden insertar las empresas en un mercado mediante innovación, no habrá incentivo alguno al lucro y mucho menos a la innovación, o al mejoramiento continuo de la calidad.

La función de aseguramiento a cargo de las EPS no se evidenció en Colombia tal como predice el modelo, en la práctica no ejercen un rol de intermediario imparcial entre la oferta y la demanda de atención en salud, por el contrario, existe una tendencia a eliminar la función de intermediación, dado que las aseguradoras cuentan con su propia red de prestadoras del servicio de salud, lo que mencionamos anteriormente como integración vertical, al menos, el primer nivel de atención, el cual, a su vez, controla los demás niveles de complejidad y tiene contacto directo con el demandante (originariamente en los arts. 156, 177, 179 y 181, Ley 100/1993). Esto lleva a que se acentúen los problemas de demanda inducida y demanda derivada, los cuales han sido motivadores de acciones judiciales en contra del funcionamiento del sistema y de posiciones divergentes por parte de quienes imparten justicia en el país (sentencias de la Corte Constitucional contradictorias en este aspecto: declarado exequible por Sentencia C-616-2001 y, posteriormente, rechazado en Sentencia T-603/2010).

Las EPS se han hecho paulatinamente propietarias de IPS para defenderse de la estrechez de los ingresos que reciben por la UPC y de la posibilidad de agremiación (oligopólica) de profesionales de la salud e IPS. También por la presencia de activos específicos por infraestructura hospitalaria, por tecnología o conocimientos especializados

\footnotetext{
${ }^{4}$ Las traducciones son nuestras.
} 
en áreas de la salud los incentivos, en este caso, tienden a "capturar rentas por la vía de bajar los costos sin importar la calidad, manteniendo las diferencias entre el gasto médico y la UPC en poder del asegurador" (Restrepo, Lopera y Rodríguez, 2007, p. 279).

Aún más, las EPS han buscado representación política por medio del lobby para conseguir hacer a su favor leyes por parte del regulador estatal, con lo cual no solo han desnaturalizado su papel de intermediario imparcial, sino que se ha desnaturalizado la imparcialidad requerida del que hace las reglas del juego del sistema: el Estado-regulador.

Todo esto se explica porque las funciones designadas al Estado parten del supuesto metodológico, que constituye un agente imparcial, informado y diferenciado de los agentes económicos del sistema (productores y consumidores), por lo cual puede regular el mercado para la mejor interacción entre ellos. Sin embargo, este es un supuesto restrictivo: el Estado no es un ente y mucho menos es imparcial, está formado por personas que también poseen intereses y actuarán conforme a ellos, por lo cual este supuesto es ingenuo, e incentiva conductas anticompetitivas y captura al regulador (Stigler, 1971). Dicho supuesto, al igual que las teorías de la década de 1960 y de la llamada vertiente de la economía pública que asume que el Estado puede actuar como empresa y regulador del mercado, "cumplir el papel de árbitro o de regulador a través de las instituciones encargadas de resolver los conflictos entre dos o más agentes" (López, 2008, p. 153), para corregir las fallas del mercado.

La figura del patrocinador que también ha estado fragmentada en manos del Fondo de Seguridad y Garantía, el Ministerio de Salud y Protección Social y la Comisión de Regulación en Salud o el Consejo Nacional de Seguridad Social desconoce que el regulador también actúa bajo cierta racionalidad, reglas e intereses (maximización de poder político o de ingresos), lo cual puede llevar a desvío de sus fines y de los sociales (colectivos). Se ha comprobado que esta regulación en Colombia ha puesto en desventaja a ciertos agentes del mercado y ha incentivado el desarrollo de procesos de corrupción y captura por grupos de presión. Un ejemplo fue el llamado caso Salucoop, en el cual Salucoop EPS, cooperativa sin ánimo de lucro, realiza un fraude al desviar los dineros de sus afiliados: "Ocho mil cheques enredan al expresidente de Saludcoop, los cuales aparecen como girados por pago de medicamentos o procedimientos que después eran presentados al Fosyga para legalizar recobros de servicios no POS” (El Tiempo, 5 abril 2012). El fallo de la Contraloría para noviembre de 2013 demuestra que el desfalco asciende a COP 1.4 billones y que su desarrollo contó con complicidad de funcionarios públicos encargados de instituciones reguladoras y administradoras de justicia.

¿Por qué los Gobiernos representan unos intereses económicos y no otros? El Gobierno está compuesto de personas (con intereses) que para llegar al poder necesitan de otras personas: los votantes (con sus propios intereses), quienes no ven muy claro cómo sus intereses (tan particulares y diferenciados unos con otros) se pueden materializar con 
un voto; no obstante, otras personas (con sus intereses) pueden persuadir con "pequeños premios inmediatos" a los votantes de dar su voto (empleo, subsidio, pagos en especie o en dinero), y esto, dependiendo del poder económico del persuasor, puede ser masivo y generalizado, por lo cual le garantiza la victoria en las elecciones a aquel que represente sus intereses. El resultado: todos los Gobiernos representan unos intereses económicos, especialmente de aquellos grupos económicos que quieren mantener o aumentar su poder económico. Si el Gobierno no tuviera la potestad de intervenir en la economía, al empresario grande o pequeño no le interesaría su particular representación para su propia ventaja y simplemente se esforzaría por ganar mercado con innovaciones, mejores precios y mejor calidad, y en consecuencia los políticos no se empeñarían en corromper el funcionamiento del Estado, las instituciones de la organización y la justicia. Si le quitamos la actividad económica al Gobierno, el único interés que representarían los políticos sería el de permitir que la actividad económica independiente de su interés fluyera y los ciudadanos podrían votar con credibilidad por aquel que con reglas generales no le obstaculice el camino para alcanzar su particular y diferenciado interés. El tema de los intereses siempre ha estado ligado al razonar económico, y olvidarlo al formular políticas es un error, más aún en el mercado de la salud que está cargado además de fuertes elementos éticos y sociales.

Las prácticas anticompetitivas generadas bajo el modelo vigente no han permitido el desarrollo de la infraestructura necesaria para responder a las diversas necesidades de salud. Por ejemplo, número de camas hospitalarias por habitante, ambulancias per capita, profesionales de la salud, entre otros, y algo marcado en Colombia: la ausencia de infraestructura en el entorno rural (Flórez, 2010b). Igualmente, es evidente la corrupción presente en el sistema, tanto de lado de la oferta como de la demanda. Se encuentra que no son solo los denominados fallos del mercado los obstáculos del sistema, sino precisamente los fallos del Estado en ejercer la función regulatoria los que han determinado rigideces en el sistema e incentivos inadecuados.

Ahora bien, además de determinar precio y producto, el otro gran factor que determina la ausencia de competencia es la imposibilidad de la demanda de elegir. En primer lugar, el sistema funciona bajo un régimen de afiliación obligatoria donde los "clientes" tienen pocas posibilidades de elegir debido al reducido número de oferentes (para 2015 trece EPS en el régimen contributivo [Ministerio de Salud y Protección Social, 2015, p. 20]) y los oferentes no tienen la posibilidad de innovar. Si existe descontento por parte del paciente, este debe esperar un tiempo (Ley 1122/2007, art. 25, par. 1: "El traslado voluntario de un usuario podrá hacerse a partir de un año de afiliado a esa EPS”) para poder trasladarse de EPS y demostrar determinado estado de salud.

Una de las reglas de Enthoven para crear un mercado competitivo en la salud es transformar el producto en un "bien normal" de la teoría microeconómica. Un bien normal denota una demanda elástica al precio, por lo cual para que la asistencia sanitaria lo 
evidencie el patrocinador debe establecer un precio estable (lowest priced) de la prima de seguro y de reaseguro. Paradójicamente, el mecanismo de precios que la teoría clásica denota se forma libremente de la interacción entre la oferta y la demanda, y dado que en este modelo se diseña por uno de los agentes o partes de la transacción, este hecho desnaturaliza la esencia del mercado.

En Colombia, el precio de producto estándar se denomina UPC. ${ }^{5}$ Para 2013, el valor de la UPC del Régimen Contributivo fue de COP 547.639. El valor de la UPC del Régimen Subsidiado fue de COP 433.666 (antes del 1 de julio el valor de la UPC era de COP 378.760). En contraste, para 2017 es de 746.046 y 667.429, respectivamente. El costo total de la unificación del POS para 2013 era de COP 120 ooo millones mensuales: "El incremento del régimen subsidiado (17.37 \%) se hace dando cumplimiento al Auto 262 de 2012 de la Corte Constitucional - sala de seguimiento de sentencia T-760 de 2008, que le ordenó al Ministerio igualar la UPC del subsidiado con el contributivo, mientras establece la suficiencia de la UPC para los dos regímenes" (Asociación Colombiana de Hospitales y Clínicas, 28 diciembre 2012). Por lo cual evidencia la contundencia política y no económica en la determinación de precios y productos del sistema de salud.

El segundo requisito para que funcione la "competencia perfecta" es diseñar deliberadamente un producto homogéneo para que sea solo este el que "el mercado" produzca. En este sentido, Enthoven introduce los denominados contratos de cobertura estandarizada, con el fin de disuadir la diferenciación de productos, facilitar la comparación de precios y calidad y evitar la segmentación del mercado por parte de los productores. La elasticidad de la demanda también se puede formar por medio de información de calidad, libre elección de los planes a nivel individual, no por grupos de trabajo (Flórez, 2010, p. 81). Sin embargo, este supuesto clásico está en contra de la naturaleza de los agentes económicos, pues, al contrario de la realidad, las ganancias y el aumento de la competencia se derivan de la innovación y de la diferenciación constante en los productos, por lo cual homogeneizarlos es un error que deriva en estancamiento del sistema y detrimento de la situación de los pacientes.

Así tenemos que tanto el precio como los productos que originalmente se conciben como resultados de la interacción de los agentes del sistema aquí se tienen como punto de origen y punto de llegada, por lo cual no se incentiva la innovación o la creación de valor por parte de los agentes productores en el sistema, sino, al contrario, se motiva el estancamiento. Pérez y Soto (2013) ha definido este fenómeno como secuestro de la información. Al eliminar la posibilidad de creación de información por medio de

\footnotetext{
${ }^{5}$ Para 2013, el valor de la UPC del Régimen Contributivo es de COP 547 639. El valor de la UPC del Régimen Subsidiado es de COP 433666 (antes del 1 de julio el valor de la UPC era de COP 378 760). El costo total de la unificación del POS es de COP 120000 millones mensuales. El incremento a partir de la unificación del POS en el valor de la UPC fue de COP 54906 millones (Así Vamos en Salud, 2017).
} 
precios y productos, se elimina también el origen de la mejora y la competitividad del mercado por la empresarialidad. El autor define el secuestro de la información o la captura de la información como

la pérdida de información en que incurre el organismo social cuando el Estado y sus representantes intentan ejecutar programas de ingeniería social, como efecto de una planificación central a gran escala. Este proceso se da por medio de la asfixia a los mecanismos de creación y transmisión de información y conocimiento, así como de sus mecanismos de contraste y comprobación. En general se puede decir que esta se sucede cuando en la ejecución de la planificación se sustituyen los modos de ordenamiento espontáneo por unos de carácter coactivo, impidiendo que en la sociedad se dé un orden autónomo, y se sustituya en su lugar por uno exógeno, devenido de la creación del sabio legislador o planificador centralizado. (Pérez y Soto, 2013, p. 26).

Este novedoso concepto puede ser complementario y derivado del propuesto por Stigler, pues, en el entorno económico con la captura del regulador, no es relevante la conducta de este per se, sino los efectos de esta en el mercado, los cuales coartan las posibilidades de generar nueva información, parcializan la interacción de los agentes hacia cierta dinámica predeterminada por la regulación capturada y eliminan las posibilidades para alcanzar la eficiencia.

El POS como mecanismo para garantizar un producto homogéneo que pudiese ser objeto de competencia presenta fallo, porque, primero, no fue un producto acorde con las necesidades epidemiológicas de la población, demostrado por la cantidad de tutelas en salud ${ }^{6}$ que demandan servicios no POS. Hasta 2010 había variado más de cuarenta veces (Actualizaciones y aclaraciones al POS-S y POS-C 1994-2010, s. f.).

Diferenciar a los usuarios por capacidad de pago, otorgar subsidios y cobrar tarifas diferenciadas origina problemas de oportunismo por parte del oferente, tales como la selección adversa, y por parte del consumidor, dado que las personas querrán, aunque su situación no lo amerite, obtener subsidios en salud, caso constante en el sistema colombiano, evidenciado en el fenómeno de la duplicidad en la afiliación por personas beneficiarias del régimen contributivo, pero con afiliación en el régimen subsidiado para obtener los beneficios del segundo.

\section{Los supuestos importan en la práctica}

Los supuestos epistemológicos han generado un diseño de regulación en el sistema de salud y con él unos efectos en la asistencia sanitaria en Colombia. En la tabla 3, se pueden

\footnotetext{
${ }^{6}$ En la primera década del siglo XXI, cerca de $30 \%$ de todas las tutelas en Colombia incoaban casos de salud y de inoportunidad del POS con las necesidades de salud (Defensoría del Pueblo, 2013).
} 
asociar los problemas enunciados en el acápite anterior con los supuestos epistemológicos que adoptó el modelo de Enthoven. Concebir el hombre como un ser enteramente calculador, pero sin elemento tiempo y, por consiguiente, sin un contexto de constante cambio y evolución lo modela como un sujeto que no aprende de los procesos ensayoerror, que no se asocia, que no aprende con el paso del tiempo y que no actúa guiado por subjetividades ocasiona la insostenibilidad y el colapso del sistema de salud colombiano.

Las EPS, dadas las reglas del sistema, han optado por cumplir con la asistencia establecida por ley, minimizando los costos y la calidad, el tiempo de consulta, la remuneración del recurso humano, la independencia profesional de este último y, lo más importante, eliminando la posibilidad de innovación y de destrucción creativa en el sistema. Al no existir precios sensibles a los movimientos de oferta y demanda, sino los dispuestos por el regulador, el cual en su fatal arrogancia (Hayek) presume establecer todas las reglas adecuadas para promover un resultado justo y eficiente, se paraliza el sentido empresarial e innovador que posibilita todo mercado.

Tabla 3. Problemas de la institucionalidad y la regulación derivados de supuestos epistemológicos

\begin{tabular}{|l|l|l|}
\hline \multicolumn{2}{|c|}{ Problema epistemológico } & \multicolumn{1}{c|}{ Problema regulatorio } \\
\hline Antropológico & $\begin{array}{l}\text { Falso individualismo }+ \\
\text { ausencia del tiempo Regulador } \\
\text { imparcial: regulador sin } \\
\text { racionalidad instrumental }\end{array}$ & Captura del regulador, corrupción, pay to play \\
\cline { 2 - 4 } & Ausencia del tiempo & $\begin{array}{l}\text { Discriminación de precios por capacidad de } \\
\text { pago: selección adversa, corrupción y free } \\
\text { riders* }\end{array}$ \\
\hline Tiempo & $\begin{array}{l}\text { Organización estática por regulación vs. } \\
\text { desempeño dinámico de los agentes } \\
\text { (integración vertical entre EPS e IPS) }\end{array}$ \\
\hline Información & $\begin{array}{l}\text { Objetivismo y pretensión de } \\
\text { información total }\end{array}$ & $\begin{array}{l}\text { Segmentación y no correspondencia del } \\
\text { producto (plan obligatorio de salud, plan } \\
\text { de beneficios en salud) con la demanda }= \\
\text { información secuestrada o capturada }\end{array}$ \\
\hline
\end{tabular}

* También conocido como polizón, agente que se aprovecha de los beneficios de una actividad sin asumir pago alguno por ella.

Fuente: Elaboración propia.

Huerta de Soto afirma que el desaparecido concepto de función empresarial en la teoría económica se refiere a "la capacidad típicamente humana para darse cuenta de las oportunidades de ganancia que surgen en el entorno actuando en consecuencia para aprovecharse de las mismas" (2004, p. 26), la regulación del sistema de salud a truncada dicha capacidad y el sistema de salud no mostrará progreso y adecuación a la demanda 
mientras no se den las libertades necesarias para garantizar su desarrollo. Solo esas libertades promoverán la competencia y la creación constante de nueva información y, de allí, la innovación y evolución del sistema.

Son estos supuestos epistemológicos los que han llevado a generalizar la creencia de que un regulador puede anticipar mejor los fines y los medios para promover cierto tipo de asistencia sanitaria. Reemplazar los fines y medios, tal como ha sucedido con los del plan obligatorio de salud o el plan de beneficios en salud, el precio del plan, la forma empresarial de aseguradoras, características o estándares para la atención, ha conllevado la anulación de un mercado competitivo de la salud y la concentración de este mercado en pocas empresas.

La regulación, función teóricamente neutral y moduladora del sistema, ha dado lugar a buscadores de renta y a la nociva relación de representantes políticos y empresarios o a la captura del regulador, accionar que, tal como sucede en la salud, llega a ser totalmente predecible, aunque aún no totalmente estudiado por la teórica económica estándar. Si bien la connotación ética puede dar lugar a otra discusión, lo que se pretende evidenciar es que el problema del diseño del sistema de salud es, precisamente, de los diseñadores, o mejor, de quienes van a ejercer el papel de su dirección, porque funcionan como empresarios con sus propios intereses y se han percatado de que obtienen ganancias extraordinarias si acuerdan pactos restrictivos de la competencia o si otorgan concesiones a los empresarios con mayor poder en el mercado.

Muchos argumentos en contra del funcionamiento actual se basan en la poca idoneidad de la privatización del sistema, se asume equivocadamente que el problema de la salud es la propiedad privada de los medios de producción y que las propuestas como las de Enthoven por tener el componente de propiedad privada en la prestación son de fundamento moral-liberal (Álvarez, 2005; Alvis, Alvis y Castro, 2009). Esto constituye un doble error, puesto que el esquema de propiedad privada es inherente a la acción económica del hombre así la financiación sea pública; el trabajo y sus frutos se derivan de la individualidad. Ahora bien, si la provisión y la financiación son privadas, pero las reglas del juego son tan restrictivas como las antes señaladas, resultan incompatibles con un esquema filosófico liberal, al cohibir las posibilidades de creación de información e innovación en pro de la mejora del sistema o de un desvío de los incentivos, haciendo que la capacidad de cálculo y la búsqueda de ganancias solo sean posibles transgrediendo la ley.

Apartar la política del funcionamiento de los sectores, como ocurre con la salud, conlleva dejar la regulación del sistema al desarrollo de la empresarialidad de la oferta de asistencia sanitaria, lo cual a su vez permitiría múltiples innovaciones para bajar los precios de los servicios: 
El acto empresarial, a la vez que coordina, crea nueva información que a su vez modifica en el mercado la percepción general de fines y medios de los actores implicados, lo cual da lugar a la aparición de nuevos desajustes, que a su vez tienden a ser descubiertos y coordinados empresarialmente, y así sucesivamente a lo largo de un proceso que jamás se detiene de expansión sin límite del conocimiento y los recursos, apoyado sobre un volumen de población siempre creciente, y que tiende a ser tan coordinado como sea humanamente posible en cada circunstancia histórica. (Huerta de Soto, 2004, p. 29)

Lo anterior muestra que el resultado final de la aplicación del managed competition es un sistema de salud ineficiente, con gran insatisfacción por parte de los usuarios, que se ven obligados a acudir a medios diferentes de los del mercado para demandar la exigibilidad de la prestación del servicio ante los tribunales de justicia, y acceder por vía judicial - acción de tutela - a aquello que el Estado les ha impedido por medio del mercado, en virtud del establecimiento coactivo de una visión planificada que niega el mercado libre. En Colombia, en la primera década del siglo XXI, las tutelas por derecho a la salud ascendieron a 26 y $27 \%$ del total de tutelas (Defensoría del Pueblo, 2013, p. 111). Asimismo, 66 \% de las tutelas por derecho a la vida contenían aspectos del derecho a la salud en contra de organizaciones, como el "Departamento para la Prosperidad Social, Coomeva, Nueva EPS, Secretarías de Salud y SaludCoop” (pp. 122-123).

El teorema de la imposibilidad del cálculo económico en el socialismo, de Ludwig von Mises, lograba mostrar que el planificador no alcanza a hacerse con la información necesaria para que los individuos hagan un cálculo económico correcto y puedan llevar a cabo las mejores decisiones en concordancia con sus intereses, precisamente, del supuesto del que parte Enthoven de perfecta información en manos del regulador. El legislador, en este caso el Congreso de la República, así como el constituyente y las Superintendencias de Salud, carecen de la información necesaria que les permita establecer un diseño institucional eficiente que logre maximizar cantidad y calidad del servicio, dentro del pretendido marco del Estado social de derecho.

Más aún, desde el concepto de información secuestrada o capturada por el regulador, puede verse que el efecto real de la legislación que impone un modo concreto de prestación del servicio fija un precio y establece coactivamente unas condiciones de idoneidad y calidad, esto es eliminar la capacidad empresarial de innovación y mejoramiento continuo. El efecto de la normativa será entonces doble. Por una parte, un efecto visible es la promoción de una organización industrial típica monopólica en la que los productores incurren en una colusión descentralizada, para la cual no requieren ponerse directamente de acuerdo en ofrecer el mínimo servicio posible, y lo hacen bajo las condiciones de calidad más bajas. Lejos de entrar en un proceso dinámico de maximización de beneficios en el que los empresarios busquen continuamente 
innovar en métodos y formas de producción cada vez más baratas y satisfactorias para los usuarios, su eje de acción será la minimización de costos. Esto en virtud de que el "mercado" se distribuye en buena medida con un criterio político, en el que la competencia se impide desde la ley, teniendo como resultado una concentración de los consumidores en unas pocas firmas, sin mayores posibilidades de movilidad, ni de reclamar efectivamente un buen servicio frente a tribunales que sancionen la conducta impropia de los productores del servicio. Por otra parte, el efecto de la legislación impide la creación de información, es decir que el legislador incurre sin saberlo en un secuestro de la información, en la captura de la capacidad de innovación, condenando a un escenario estático de insatisfacción a los usuarios ad infinitum.

La pretensión del legislador de establecer un diseño del mundo social "por decreto" choca con la realidad de que el mercado logre satisfacer las necesidades de las personas de la mejor manera posible, en virtud de la libertad individual que conlleva un continuo afán por mejorar la situación personal, como la vocación empresarial, lo cual a su vez invita a la continua realización de microexperimentos en busca de nuevas y mejores formas de hacer y de refinar los modos de producción. Al ampliar este proceso a miles de empresarios, se puede entender cómo el avance social no corresponde a la sabiduría de un legislador, sino al conocimiento descentralizado, subjetivo, abstracto, parcialmente transferible y en continuo perfeccionamiento que puedan proveer los empresarios. Limitar el mercado, el producto, el precio, su idoneidad, calidad y características intrínsecas no logrará prestar el mejor servicio posible, lejos de esto, estará bloqueando el mecanismo por medio del cual los consumidores cada vez pueden acceder a un mejor servicio, en virtud de una auténtica competencia. No la del modelo neoclásico, sino una competencia verdadera, con diferencias en la información, con existencia de externalidades y de poderes de mercado que se pueden vencer en el mismo momento en que un productor sea capaz de producir mejor y más barato que sus competidores.

\section{Conclusión}

Desde la perspectiva de la escuela austriaca de economía, la competencia es un proceso de descubrimiento de los agentes, que permite innovar en formas y métodos de producción, el cual no es predecible, ni mucho menos diseñado, por un planificador. El problema del diseño del sistema de salud debe asumirse partiendo del error y la incertidumbre humanas: "La concepción de una mente plenamente desarrollada que diseña las instituciones que hacen posible la vida en sociedad es contraria a cuanto sabemos sobre la evolución del hombre" (Hayek, 2006, p. 36). Por ello, el regulador debería realizar su tarea confiando en que tener reglas generales para la acción libre, evolutiva y creativa, y no mandatos específicos para actuar de cierta manera, es la fórmula para potenciar la producción y el intercambio de mejor calidad. 
La reforma de salud no se puede realizar si no se parte de fundamentos epistemológicos y metodológicos cercanos a la realidad. Una vez se interiorice en los analistas y los policy makers esta concepción, se podrá disponer de mejores medios para alcanzar los fines deseados. El razonamiento a partir de este nuevo modelo, y la deducción de conclusiones, conducirá a un contexto de no intervención en el modo y el desarrollo de los intercambios humanos y a una reevaluación de las funciones del Estado para garantizarlos. Esto no quiere decir que se prefiera un contexto anárquico, sino, al contrario, uno en que el Estado funcione como garante y vigilante de que los acuerdos y las normas emanados del desarrollo espontáneo de los intercambios se cumplan y exijan. El Estado debe ser un potenciador del desarrollo humano y no un obstáculo para este:

El argumento a favor de la libertad no es un argumento contra la organización, uno de los más poderosos medios que la razón humana puede utilizar, sino contra todas las organizaciones exclusivas, privilegiadas y monopolísticas, contra el uso de la coacción para impedir a otros que traten de hacerlo mejor. (Hayek, 1997, p. 56)

Se deja abierto el debate para que se piense en un sistema de asistencia sanitaria desde una posición epistemológica que incluya algunos de los elementos propuestos por la escuela austriaca, que pudieran ser beneficiosos para el sistema de salud colombiano, tales como empresarialidad natural (Huerta de Soto, 2004), información dispersa (Hayek, 1997), racionalidad limitada (Hayek, 2007) e información secuestrada (Pérez y Soto, 2013). Un cambio en el sistema de salud que contribuya a garantizar el derecho a la salud demanda un diseño institucional basado en supuestos más cercanos al comportamiento de las personas e incentivos para la acción de los agentes del sistema.

\section{Referencias}

Actualizaciones y aclaraciones al POS-S y POS-C 1994-2010. Recuperado de http://www. med-informatica.net/FMC_CMCB/VeeduriaCiudadana/CIDMEDvcacelapss/ MPS_ActualizacionesAclaracionesPOS-SyPOS-Cde1994a2010.pdf

Álvarez Castaño, L. S. (2005). El derecho a la salud en Colombia: una propuesta para su fundamentación moral. Revista Panamericana de Salud Pública, 18(2), 129-135.

Alvis, N., Alvis, L. y Castro, R. (2009). Inequidad regional en la financiación de la salud en Colombia. Lecturas de Economía, 71, 169-188.

Arrow, K. J. (1963). Uncertainty and the welfare economics of medical care. American Economic Review, 53(5), 941-973.

Asociación Colombiana de Hospitales y Clínicas (28 diciembre 2012). Minsalud aprobó incremento de la UPC para 2013. Recuperado de http://www.achc.org.co/ noticias.php?idnoticia $=51$ 
Así Vamos en Salud (2017). Evolución de la UPC en Colombia. Recuperado de https:// www.asivamosensalud.org/indicadores/financiamiento/evolucion-de-la -upc-nominal-en-pesos-corrientes

Banco Mundial (2015). Gasto en salud, total (\% del PIB). Recuperado de https://datos. bancomundial.org/indicador/SH.XPD.TOTL.ZS

Calderón, J. (2015). Autonomía médica y ley estatutaria de salud. Acta Médica Colombiana, 4O(1), 51-53.

Collazo Herrera, M., Cárdenas Rodríguez, J., González López, R., Miyar Abreu, R., Gálvez González, A. M. y Cosme Casulo, J. (2002). La economía de la salud: ¿debe ser de interés para el campo sanitario? Revista Panamericana de Salud Pública, 12(5), 359-365.

Congreso de la República. Ley 100 de 1993. Por la cual se crea el sistema de seguridad social integral y se dictan otras disposiciones (23 diciembre 1993).

Corte Constitucional. Sentencia C-616/2001. Seguridad social.

Corte Constitucional. Sentencia T-603/2010. Derecho a la libre escogencia de IPS por parte del usuario.

Defensoría del Pueblo (2013). La tutela y el derecho a la salud 2012. Bogotá: Defensoría del Pueblo.

El Espectador (25 febrero 2013). Proponen abolir del sistema de salud la 'cultura del acetaminofén'. Recuperado de https://www.elespectador.com/noticias/politica/ proponen-abolir-del-sistema-de-salud-cultura-del-acetam-articulo-406763

El Tiempo (5 abril 2012). Ocho mil cheques enredan a Carlos Palacino, el 'cerebro' de Saludcoop. Recuperado de http://www.eltiempo.com/archivo/documento/ CMS-11506840

Enthoven, A. C. (1993). The history and principles of managed competition. Health Affairs, 12(1), 24-48.

Flórez Pinilla, K. (2008). Prestación de servicios de salud en Colombia: dilema entre el Estado y el mercado. IUSTITIA, 6, 163-182.

Flórez Pinilla, K. (2010a). La competencia regulada en el sistema de salud colombiano. Revista Lebret, 2, 73-99.

Flórez Pinilla, K. (2010b). Tensiones institucionales en el sistema de salud colombiano. IUSTITIA, 8, 271-296.

Hayek, F. A. (1997). Los fundamentos de la libertad. Barcelona: Folio. 
Hayek, F. A. (1999). El uso del conocimiento en la sociedad. Cuadernos de Economía, 18(30), 331-345.

Hayek, F. A. (2006). Derecho, legislación y libertad. Madrid: Unión Editorial.

Hayek, F. A. (2007). Individualismo: el verdadero y el falso. Madrid: Unión Editorial.

Health Consumer Powerhouse (2017). Euro Health Consumer Index 2016. 2017-01-30.

Huerta de Soto, J. (2004). La teoría de la eficiencia dinámica. Procesos de Mercado: Revista Europea de Economía Política, 1(1), 11-71.

Huerta de Soto, J. (2005). Socialismo, cálculo económico y función empresarial (3. ${ }^{\mathrm{a}}$ ed.). Madrid: Unión Editorial.

Londoño, J. L. y Frenk, J. (1997). Pluralismo estructurado: hacia un modelo innovador para la reforma en los sistemas de salud en América Latina. Working Paper, Inter-American Development Bank, Office of the Chief Economist 353.

López Pontón, E. (2008). Un criterio de eficiencia para la concepción y la evaluación de las políticas públicas. Revista de Economía Institucional, 10(18), 9-178.

Matajira Gaitán, C. A., Escobar Morales, G. y Barney Iglesias, E. A. (2016). Sistema de evaluación y calificación de actores: ranking de satisfacción EPS 2016. Bogotá: Oficina de Calidad, Ministerio de Salud y Protección Social y Protección Social.

Ministerio de Salud y Protección Social (2015). Ordenamiento por desempeño EPS 2015. Bogotá: Ministerio de Salud y Protección Social.

Mushkin, S. J. (1999). Hacia una definición de la economía de la salud. Lecturas de Economía, 51, 89-109.

OPS/OMS (Organización Panamericana de la Salud/Organización Mundial de la Salud) (2011). Economía y salud: aportes y experiencias en América Latina. Santiago de Chile: OPS/OMS.

Pérez y Soto Domínguez, A. (2013). La información secuestrada: el modelo de servidumbre de Thomas Hobbes y su alternativa liberal austriaca en Friedrich Hayek (Tesis doctoral, Universidad Rey Juan Carlos, Madrid, España).

Restrepo, J. H., Lopera, J. F. y Rodríguez, S. M. (2007). La integración vertical en el sistema de salud colombiano. Revista de Economía Institucional, 9(17), 279-308.

Stigler, G. J. (1971). The theory of economic regulation. The Bell Journal of Economics and Management Science, 2(1), 3-21. 